TU-785

March, 2007

\title{
Big-Bang Nucleosynthesis with Long-Lived Charged Slepton
}

\author{
${ }^{(a)}$ Masahiro Kawasaki, ${ }^{(b)}$ Kazunori Kohri, and ${ }^{(c)}$ Takeo Moroi \\ ${ }^{(a)}$ Institute for Cosmic Ray Research, University of Tokyo, Kashiwa 277-8582, JAPAN \\ (b) Physics Department, Lancaster University, Lancaster LA1 4YB, UK \\ (c) Department of Physics, Tohoku University, Sendai 980-8578, JAPAN
}

\begin{abstract}
We consider constraints on long-lived charged scalar leptons $\tilde{l}^{ \pm}$in supersymmetric models, where gravitino is the lightest superparticle. We study the decay and hadronization processes of $\tilde{l}^{ \pm}$. We also take into account the significant enhancement of ${ }^{6} \mathrm{Li}$ production due to the formation of the bound-state $\left({ }^{4} \mathrm{He} \tilde{l}^{-}\right)$; for this purpose, we use the reaction rate given by the most recent calculation based on coupled-channel method.
\end{abstract}


Cosmology provides important constraints on physics beyond the standard model. One of the important examples is constraints on long-lived particles from big-bang nucleosynthesis $(\mathrm{BBN})$; since the theoretical predictions of the standard BBN scenario are in reasonable agreements with observation of light-element abundances, we obtain constraints on longlived particles whose decay may induce photo- and hadro-dissociation processes.

Here, as a physics beyond the standard model, we consider low energy supersymmetry (SUSY), which is strongly motivated as a prominent solution to serious problems in the standard model, like the hierarchy problem, the dark-matter problem, and so on. In the framework of SUSY models, we assume that one of the charged sleptons is the lightest particle among the superpaticle in the minimal-supersymmetric-standard-model (MSSM) sector, and that the gravitino is the lightest superparticle (LSP). Such a mass spectrum is easily realized; probably the most famous example is gauge-mediated SUSY breaking models [1]. Thus, such a scenario has been attracted much attention, in particular, in the context of gravitino LSP [2, 3, 4, 15, 6, 7, , 8, 9]. Then, the lightest slepton $\tilde{l}$ is usually long-lived and the decay of $\tilde{l}$ may spoil the success of BBN. Indeed, the BBN scenario with long-lived charged sleptons has been discussed in several works [6, 7, 10].

In this letter, we reconsider effects of long-lived charged slepton on BBN. We perform a detailed study on the decay processes of slepton and the hadronization processes of the decay products. In addition, we also take into account the effect of bound-state formation; recently, it has been pointed out that long-lived charged slepton may form bound states with light elements [11, 12, 13], which may significantly change the BBN reaction rates [11]. In particular we take a serious attitude towards the bound-state effect by $\left({ }^{4} \mathrm{He} \tilde{l}^{-}\right)$pointed out by [11] (which is called $\tilde{l}^{-}$-catalyzed process) and include it into our analysis, taking into account the result of the most recent precise calculation of the bound-state effect [14.

Let us first summarize the basic properties of the lightest slepton $\tilde{l}$ and the gravitino (which is denoted as $\psi_{\mu}$ hereafter). Since the gravitino is the only superparticle lighter than $\tilde{l}$, the dominant decay mode of $\tilde{l}$ is $\tilde{l} \rightarrow l \psi_{\mu}$. The decay rate for this process is given by

$$
\Gamma_{\tilde{l}}=\frac{m_{\tilde{l}}^{5}}{48 \pi m_{3 / 2}^{2} M_{*}^{2}}\left(1-\frac{m_{3 / 2}^{2}}{m_{\tilde{l}}^{2}}\right)^{4},
$$

where $m_{\tilde{l}}$ and $m_{3 / 2}$ are slepton mass and gravitino mass, respectively, and $M_{*} \simeq 2.4 \times$ $10^{18} \mathrm{GeV}$ is the reduced Planck scale. When the gravitino is much lighter than $\tilde{l}$, we obtain

$$
\tau_{\tilde{l}} \simeq 5.7 \times 10^{4} \mathrm{sec} \times\left(\frac{m_{\tilde{l}}}{100 \mathrm{GeV}}\right)^{-5}\left(\frac{m_{3 / 2}}{1 \mathrm{GeV}}\right)^{2} .
$$

For the case where the next-to-the-lightest superparticle (NLSP) is selectron $\tilde{e}$, the daughter particles are stable. For other cases, on the contrary, the final-state lepton also decays; such decay process is sometimes important. In particular, when stau is the NLSP, $\tau$-lepton in the final state decays subsequently and produces pion(s). As we will discuss later, such pions become a source of $p \leftrightarrow n$ conversion. In addition, when $\mu$-and $\tau$-lepton decays, neutrinos carry away some amount of energy, which affects the total amount of visible energy emitted by the decay of $\tilde{l}$. 
In our analysis, we study the decay processes of unstable leptons (in particular, that of $\tau$-lepton) using PYTHIA package [15] and derive energy distributions of the decay products. For the decay of $\tau$-lepton, for example, the averaged number of charged pions produced by the decay of $\tau$-lepton is 0.94 , which changes the ${ }^{4} \mathrm{He}$ abundance as we will see later. With the result given by PYTHIA analysis, we also calculate the (averaged) visible energy emitted from $\tilde{l}$ taking into account the energy loss due to the neutrino emission.

Charged sleptons may also decay into 3- and/or 4-body final states as $\tilde{l} \rightarrow l \psi_{\mu} Z^{(*)}$, followed by the decay of $Z^{(*)} \#$ Although 3- and 4-body decay processes are sub-dominant, they are important for the study of hadro-dissociation processes. When $Z^{(*)}$ decays into $q \bar{q}$ pair, protons, neutrons, and pions are emitted after hadronizing final-state quarks. These hadrons induce hadro-dissociation and $p \leftrightarrow n$ conversion processes, which affect abundances of light elements. (For details, see, for example, [16].) In order to study the effects of hadrodissociation and $p \leftrightarrow n$ conversion processes, it is necessary to know the hadronic branching ratio of $\tilde{l}$ as well as the spectra of final-state hadrons. We have also calculated the decay rate for the processes $\tilde{l} \rightarrow l \psi_{\mu} q \bar{q}$. Detailed information about the 3- and 4-body decay processes can be found in [10]; we found that our results are consistent with those shown in [10]. We have also calculated the invariant-mass and energy distributions of final-state quark pair. Then, using PYTHIA package, we calculate energy distributions of $p, n$, and $\pi^{ \pm}$.

Once the decay rate, total visible energy emitted by the decay of $\tilde{l}$, and spectra of $p, n$ and $\pi^{ \pm}$are obtained, we calculate the light-element abundances taking account of photoand hadro-dissociation processes as well as $p \leftrightarrow n$ conversion processes:\#2 we follow the procedure given in [16] in order to calculate the abundances of $\mathrm{D},{ }^{3} \mathrm{He},{ }^{4} \mathrm{He},{ }^{6} \mathrm{Li}$ and ${ }^{7} \mathrm{Li}$ for processes except for $\left({ }^{4} \mathrm{He} \tilde{l}^{-}\right)+\mathrm{D} \rightarrow{ }^{6} \mathrm{Li}+\tilde{l}^{-}$.

One of the important new ingredients in the current study is the $\tilde{l}^{-}$-catalyzed process for ${ }^{6} \mathrm{Li}$ production [11] \#3 In the standard $\mathrm{BBN}$ scenario, the production rate of ${ }^{6} \mathrm{Li}$ is very tiny because the $\mathrm{E} 1$ transition process for $\mathrm{D}\left({ }^{4} \mathrm{He}, \gamma\right){ }^{6} \mathrm{Li}$ process is extremely suppressed, resulting in very small primordial abundance of ${ }^{6} \mathrm{Li}$ which is consistent with observation. Recently, however, it was claimed that the ${ }^{6} \mathrm{Li}$ abundance may become much larger than the standard BBN prediction if there exists a long-lived heavy charged particle [11]. This is due to the enhancedment of the process

$$
\left({ }^{4} \mathrm{He} \tilde{l}^{-}\right)+\mathrm{D} \rightarrow{ }^{6} \mathrm{Li}+\tilde{l}^{-},
$$

where $\left({ }^{4} \mathrm{He} \tilde{l}^{-}\right)$denotes the bound state formed by ${ }^{4} \mathrm{He}$ and $\tilde{l}^{-}$. Importantly, for this process, accurate calculation for the reaction rate is performed with the use of coupled-channel method which is known to accurately describe other three-body systems [14]. Thus, we adopt the results of [14] and take into account the bound-state effect in the calculation of ${ }^{6} \mathrm{Li}$ abundance.

${ }^{\# 1}$ Here, we assume that the sleptons are right-handed. In such a case, 3- and 4-body decay processes via the emission of $W^{ \pm}$are irrelevant.

${ }^{\# 2}$ We have omitted contributions from Kaons as discussed in [16].

${ }^{\# 3}$ See also [12, 13] for recent progress in this field. 
In order to estimate the number density of $\left({ }^{4} \mathrm{He} \tilde{l}^{-}\right)$bound state $n_{\mathrm{BS}}$, we solve the Sahatype equation [17]\#4

$$
n_{\mathrm{BS}}=\left(\frac{m^{4} \mathrm{He} T}{2 \pi}\right)^{-3 / 2} e^{E_{\mathrm{B}} / T}\left(n_{4} \mathrm{He}-n_{\mathrm{BS}}\right)\left(n_{\tilde{l}^{-}}-n_{\mathrm{BS}}\right),
$$

where $E_{\mathrm{B}} \simeq 337.33 \mathrm{keV}$ [14] is the binding energy, and $n_{4}{ }_{\mathrm{He}}$ and $n_{\tilde{l}^{-}}$are total number densities of ${ }^{4} \mathrm{He}$ and $\tilde{l}^{-}$(which include those in the bound state), respectively. (In this letter, $n_{X}$ is used for the number density of particle $X$.) Here, we assume that the process ${ }^{4} \mathrm{He}+\tilde{l}^{-} \leftrightarrow\left({ }^{4} \mathrm{He} \tilde{l}^{-}\right)$is in chemical equilibrium. Then, the total reaction rate $R$ for ${ }^{6} \mathrm{Li}$ production by $\mathrm{D}-{ }^{4} \mathrm{He}$ collision is calculated as

$$
R=\frac{n_{\mathrm{BS}}}{n_{4} \mathrm{He}} R_{\mathrm{BS}}+\left(1-\frac{n_{\mathrm{BS}}}{n_{4} \mathrm{He}}\right) R_{\mathrm{SBBN}}
$$

where $R_{\mathrm{BS}}$ is given by Eq. (4.3) in [14] and $R_{\mathrm{SBBN}}$ is the standard-BBN value.

Once the light-element abundances are theoretically calculated, we compare them with observational constraints and derived $95 \%$ C.L. constraints. As observational constraints on the primordial abundances of light elements, we adopt those used in [19] except for $Y_{\mathrm{p}}$ and $\left(n_{3} \mathrm{He} / n_{\mathrm{D}}\right)_{\mathrm{p}}$ :

$$
\begin{aligned}
\left(n_{\mathrm{D}} / n_{\mathrm{H}}\right)_{\mathrm{p}} & =(2.82 \pm 0.26) \times 10^{-5} \\
\left(n_{3 \mathrm{He}} / n_{\mathrm{D}}\right)_{\mathrm{p}} & <0.83+0.27 \\
Y_{\mathrm{p}} & =0.2516 \pm 0.0040 \\
\log _{10}\left(n_{7} \mathrm{Li} / n_{\mathrm{H}}\right)_{\mathrm{p}} & =-9.63 \pm 0.06 \pm 0.3 \\
\left(n_{6} \mathrm{Li} / n_{7} \mathrm{Li}\right)_{\mathrm{p}} & <0.046 \pm 0.022+0.084
\end{aligned}
$$

where the subscript "p" is for primordial value (just after BBN), and $Y_{\mathrm{p}}$ is the primordial mass fraction of ${ }^{4} \mathrm{He}$. For the center value of $Y_{\mathrm{p}}$, we have adopted a quite recent value reported in [20] in which the authors used new data of HeI emissivites, \#5 and conservatively added larger errors of 0.0040 as shown in [22]. For $\left(n_{3} \mathrm{He} / n_{\mathrm{D}}\right)_{\mathrm{p}}$ we have used most newly-reported values of $\mathrm{D}$ and ${ }^{3} \mathrm{He}$ abundances observed in the protosolar cloud [23].

Now, we are at the position to show our results. First, we consider the case where there is no entropy production after the freeze out of $\tilde{l}$. In this case, the abundance of $\tilde{l}$ is thermally determined; then, for right-handed slepton, the yield variable (which is defined as $Y_{\tilde{l}} \equiv\left[n_{\tilde{l}} / s\right]_{t \ll \tau_{\tilde{l}}}$, with $s$ being entropy density) is estimated as $[24$

$$
Y_{\tilde{l}} \simeq 7 \times 10^{-14} \times\left(\frac{m_{\tilde{l}}}{100 \mathrm{GeV}}\right)
$$

Notice that, in the solving Eq. (4), $n_{\tilde{l}^{-}}$is given by

$$
n_{\tilde{l}^{-}}=\frac{1}{2} s Y_{\tilde{l}} e^{-t / \tau_{\tilde{l}}} .
$$




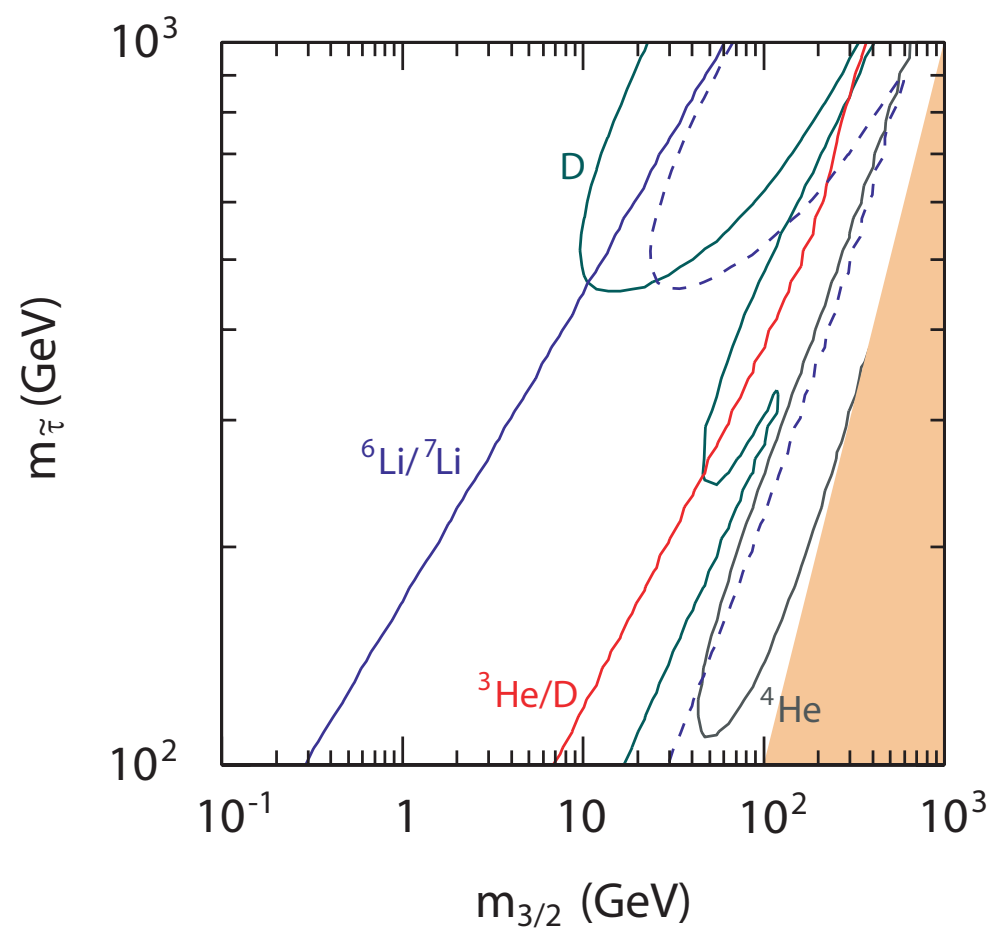

Figure 1: Constraints on $m_{3 / 2}$ vs. $m_{\tilde{\tau}_{R}}$ plane. The dashed line indicates the constraint from $\left(n_{6} \mathrm{Li} / n_{7} \mathrm{Li}\right)_{\mathrm{p}}$ without taking account of the $\tilde{l}^{-}$-catalyzed process. We have shaded the region where $\tilde{\tau}_{R}$ becomes lighter than gravitino.

since $n_{\tilde{l}^{-}}=n_{\tilde{l}^{+}}=0.5 n_{\tilde{l}}$.

With the thermal abundance, we perform our analysis for the case where $\tilde{l}$ is $\tilde{\tau}_{R}$. The result is shown in Fig. 1. For comparison, we also show the result without including the $\tilde{l}^{-}$-catalyzed process (3). As expected, constraints from light elements other than ${ }^{6} \mathrm{Li}$ is unaffected by the inclusion of the $\tilde{l}^{-}$-catalyzed process (3); those constraints are more or less consistent with the results obtained in earlier analysis [6, 7, 10]. As one can see, however, significant amount of the parameter region is excluded with the overproduction of ${ }^{6} \mathrm{Li}$ once the $\tilde{l}^{-}$-catalyzed process is considered. For the case where $m_{\tilde{l}}=100 \mathrm{GeV}$, for example, the upper bound on the gravitino mass becomes $\sim 0.2 \mathrm{GeV}$, which was $\sim 7 \mathrm{GeV}$ before the process (3) is taken into account.

So far, we have assumed that the primordial abundance of $\tilde{l}$ is thermally determined. With non-standard evolution of the universe, however, $Y_{\tilde{l}}$ may deviate from the thermal value given in Eq. (11). Thus, we also treat the primordial abundance of $\tilde{l}$ as a free parameter and derive upper bound on $Y_{\tilde{l}}$. For $\tilde{\tau}_{R^{-}}$NLSP, the result is shown in Fig. 2, taking $m_{\tilde{\tau}_{R}}=100 \mathrm{GeV}$. As one can see, the $\tilde{l}^{-}$-catalyzed process significantly changes the upper bound; for $m_{\tilde{\tau}_{R}}=$

\footnotetext{
${ }^{\# 4}$ For more detailed discussion, see [12, 18].

${ }^{\# 5}$ See also recent value of $Y_{\mathrm{p}}$ reported in [21] where the authors adopted larger errors (0.0028) than that of [20].
} 


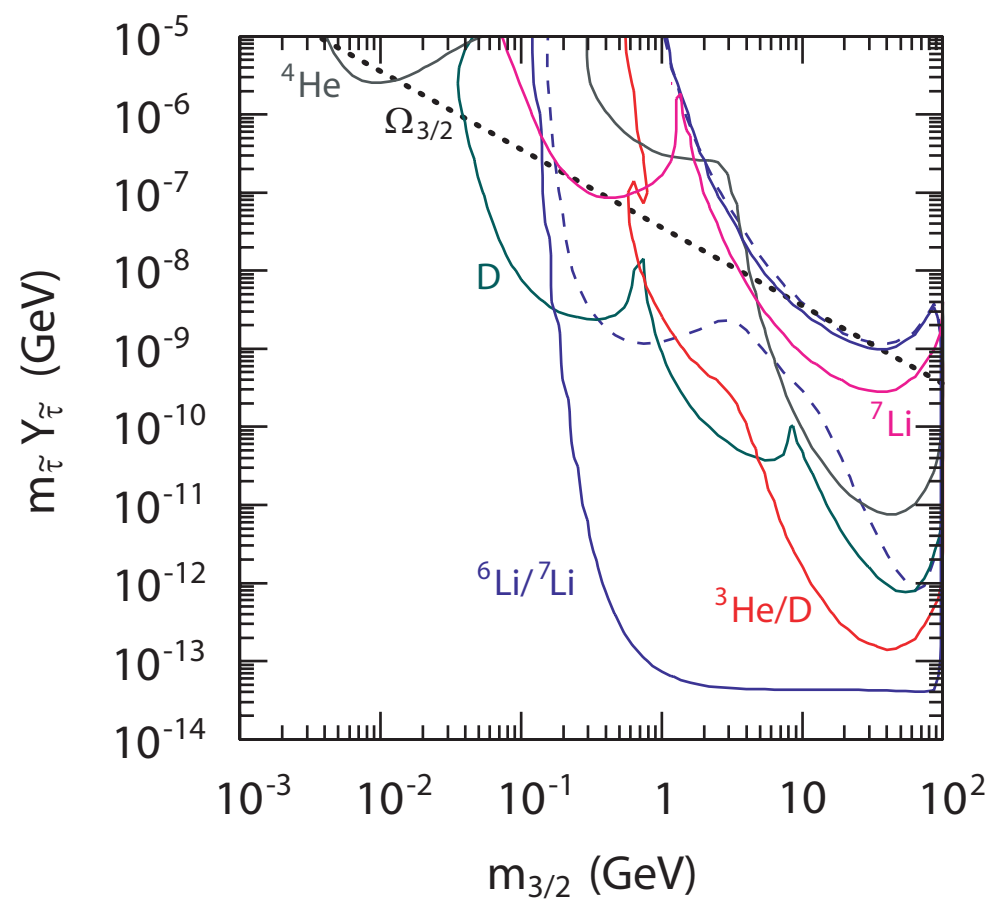

Figure 2: Upper bounds on $m_{\tilde{\tau}_{R}} Y_{\tilde{\tau}_{R}}$ as functions of $m_{3 / 2}$ for $m_{\tilde{\tau}_{R}}=100 \mathrm{GeV}$. The solid lines are bounds from various light-element abundances, while the dashed line is the upper bound from $\left(n_{6 \mathrm{Li}} / n_{7 \mathrm{Li}}\right)_{\mathrm{p}}$ without taking account of the $\tilde{l}^{-}$-catalyzed process. Thick dotted line shows the contour where the density parameter of the gravitino produced by the decay of stau becomes as large as that of cold dark matter.

$100 \mathrm{GeV}$, for example, the very stringent constraint is obtained when the gravitino mass is larger than $O(0.1 \mathrm{GeV})$, which corresponds to $\tau_{\bar{l}} \gtrsim O\left(10^{3} \mathrm{sec}\right)$. We have also considered the case with $m_{\tilde{\tau}_{R}}=300 \mathrm{GeV}$, and the result is shown in Fig. 3. For $m_{\tilde{\tau}_{R}}=300 \mathrm{GeV}$, the constraints from the hadro-dissociation processes are more stringent than those for $m_{\tilde{\tau}_{R}}=$ $100 \mathrm{GeV}$. This is mainly because, for the case of $m_{\tilde{\tau}_{R}}=300 \mathrm{GeV}$, the decay mode $\tilde{\tau}_{R} \rightarrow \tau Z$ becomes more effective, resulting in larger number of $p$ and $n$.

We have also studied another cases that the NLSP is the right-handed selectron $\tilde{e}_{R}$. In this case, the constraints slightly change. First, as we mentioned earlier, in the dominant decay mode, $\tilde{e}_{R}$ decays into gravitino and electron, which are both stable. Thus, contrary to the $\tilde{\tau}_{R}$-case, the pion production is only via three- and/or four-body decay processes, and hence is significantly suppressed. Consequently, the constraint from the $p \leftrightarrow n$ conversion is weakened. Second, the visible energy emitted by the slepton decay is larger for the selectronNLSP case since there is no energy loss due to the neutrino emission (in the two-body decay process). As a result, the constraint from the photo-dissociation process becomes more stringent than the stau-NLSP case. We checked that the upper bound on $Y_{\tilde{l}}$ from the ratio $\left(n_{3 \mathrm{He}} / n_{\mathrm{D}}\right)_{\mathrm{p}}$ becomes smaller by factor $\sim 3$ compared to the stau-NLSP case.

Finally, we comment on the bound-state effects on other processes. In our analysis, we 


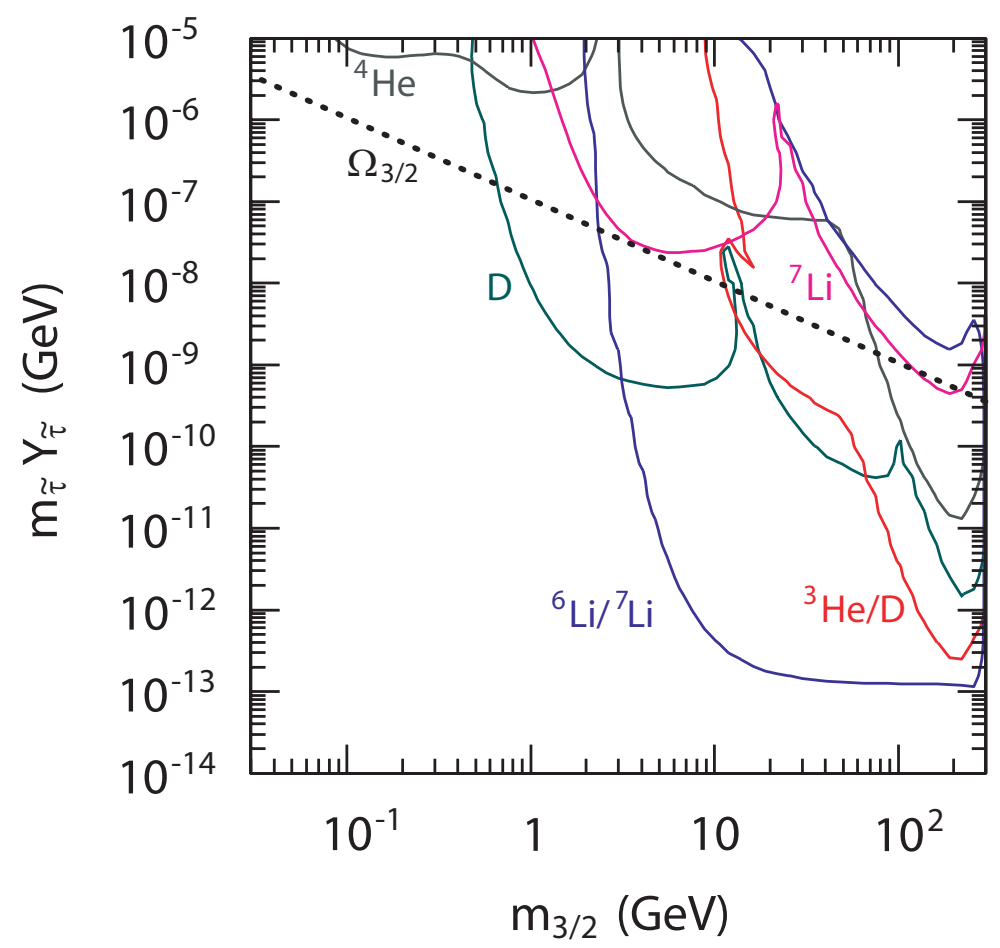

Figure 3: Same as Fig. 2, except for $m_{\tilde{\tau}_{R}}=300 \mathrm{GeV}$.

consider only the $\tilde{l}^{-}$-catalyzed process for the ${ }^{6} \mathrm{Li}$ production. In fact, reaction rates of other processes for which the E1 transition is effective (like $\mathrm{T}\left({ }^{4} \mathrm{He}, \gamma\right){ }^{7} \mathrm{Li}$ and ${ }^{3} \mathrm{He}\left({ }^{4} \mathrm{He}, \gamma\right){ }^{7} \mathrm{Be}$ ) may be also enhanced by the bound-state formation. Unfortunately, for those processes, we could not find reliable calculation of the reaction rates. However, we expect that the constraint from ${ }^{7} \mathrm{Li}$ is less stringent than that from ${ }^{6} \mathrm{Li}$ even if the bound-state effect is taken into account for ${ }^{7} \mathrm{Li}$ and ${ }^{7} \mathrm{Be}$ productions; there are several reasons why we consider so. First, observationally, ${ }^{6} \mathrm{Li}$ is rarer than ${ }^{7} \mathrm{Li}$, as one can see in Eq. (10). Thus, the constraint on the model is usually much more sensitive to the change of ${ }^{6} \mathrm{Li}$ rather than that of ${ }^{7} \mathrm{Li}$. In addition, reaction rates for the processes $\mathrm{T}\left(\left({ }^{4} \mathrm{He} l^{-}\right), l^{-}\right)^{7} \mathrm{Li}$ and ${ }^{3} \mathrm{He}\left(\left({ }^{4} \mathrm{Hel} l^{-}\right), l^{-}\right){ }^{7} \mathrm{Be}$ should acquire extra suppressions compared to the process (3); for the formar, one should keep in mind that the number density of $\mathrm{T}$ during $\mathrm{BBN}$ is about two orders of mangnitude smaller than that of D while, for the latter, the Coulomb suppression becomes much more significant compared to the process (3). Furthermore, we have also performed numerical calculation by using the enhancement factor presented in [11] for ${ }^{7} \mathrm{Li}$ and ${ }^{7} \mathrm{Be}$ productions. (Notice that, for ${ }^{6} \mathrm{Li}$ production process (3), the formula given in [11] predicts reaction rate which is one order of magnitude larger than the result of the detailed calculation given in [14].) Then we have checked that, even with these modifications of reaction rates, constraint from ${ }^{7} \mathrm{Li}$ is much less stringent than that from ${ }^{6} \mathrm{Li}$. Thus, we expect that the bound-state effects on the ${ }^{7} \mathrm{Li}$ and ${ }^{7} \mathrm{Be}$ production processes do not change our main conclusion.

Note Added: After the completion of this work, we found the paper [25] which discusses 
possible effects of the formation of bound states $\left({ }^{7} \mathrm{Li} \tilde{l}^{-}\right)$and $\left({ }^{7} \mathrm{Be} \tilde{l}^{-}\right)$. Such bound states, however, do not have significant effect on ${ }^{6} \mathrm{Li}$ formation and hence our main conclusion is unchanged.

Acknowledgements: This work was supported in part by PPARC grant, PP/D000394/1, EU grant MRTN-CT-2006-035863, the European Union through the Marie Curie Research and Training Network "UniverseNet" (MRTN-CT-2006-035863) (KK).

\section{References}

[1] M. Dine, A. E. Nelson and Y. Shirman, Phys. Rev. D 51 (1995) 1362; M. Dine, A. E. Nelson, Y. Nir and Y. Shirman, Phys. Rev. D 53 (1996) 2658.

[2] T. Moroi, H. Murayama and M. Yamaguchi, Phys. Lett. B 303 (1993) 289.

[3] J. L. Feng, A. Rajaraman and F. Takayama, Phys. Rev. Lett. 91 (2003) 011302.

[4] J. L. Feng, A. Rajaraman and F. Takayama, Phys. Rev. D 68 (2003) 063504.

[5] J. R. Ellis, K. A. Olive, Y. Santoso and V. C. Spanos, Phys. Lett. B 588 (2004) 7.

[6] J. L. Feng, S. f. Su and F. Takayama, Phys. Rev. D 70 (2004) 063514.

[7] J. L. Feng, S. Su and F. Takayama, Phys. Rev. D 70 (2004) 075019.

[8] L. Roszkowski, R. Ruiz de Austri and K. Y. Choi, JHEP 0508 (2005) 080.

[9] D. G. Cerdeno et al., JCAP bf 0606 (2006) 005.

[10] F. D. Steffen, JCAP 0609 (2006) 001.

[11] M. Pospelov, arXiv:hep-ph/0605215.

[12] K. Kohri and F. Takayama, arXiv:hep-ph/0605243.

[13] M. Kaplinghat and A. Rajaraman, Phys. Rev. D 74, 103004 (2006); R. H. Cyburt, J. Ellis, B. D. Fields, K. A. Olive and V. C. Spanos, JCAP 0611, 014 (2006); F. D. Steffen, arXiv:hep-ph/0611027.

[14] K. Hamaguchi, T. Hatsuda, M. Kamimura, Y. Kino and T. T. Yanagida, arXiv:hep-ph/0702274.

[15] T. Sjostrand, S. Mrenna and P. Skands, JHEP 0605 (2006) 026.

[16] M. Kawasaki, K. Kohri and T. Moroi, Phys. Lett. B 625, 7 (2005); Phys. Rev. D 71, 083502 (2005).

[17] See, for example, E. W. Kolb and M. S. Turner, Front. Phys. 69 (1990) 1. 
[18] K. Kohri and F. Takayama, in preparation.

[19] T. Kanzaki, M. Kawasaki, K. Kohri and T. Moroi, Phys. Rev. D 75 (2007) 025011.

[20] Y. I. Izotov, T. X. Thuan and G. Stasinska, arXiv:astro-ph/0702072.

[21] M. Peimbert, V. Luridiana and A. Peimbert, arXiv:astro-ph/0701580.

[22] M. Fukugita and M. Kawasaki, Astrophys. J. 646 (2006) 691.

[23] J. Geiss and G. Gloeckler, Space Sience Reviews 106 (2003) 3.

[24] M. Fujii, M. Ibe and T. Yanagida, Phys. Lett. B 579 (2004) 6.

[25] C. Bird, K. Koopmans and M. Pospelov, arXve:hep-ph/0703096. 